\title{
Chaos suppression of a subsonic panel with geometric nonlinearity based on Melnikov's method
}

\author{
Huaiping Tang · Peng Li · Yiren Yang
}

Received: 22 September 2013 / Revised: 18 November 2013 / Accepted: 22 November 2013 / Published online: 10 December 2013

(C) Springer-Verlag Berlin Heidelberg 2013

\begin{abstract}
Melnikov's method was adopted to study chaotic vibrations and chaos suppression of a two-dimensional subsonic panel with geometric nonlinearity subjected to external excitation. A Galerkin-type solution was introduced to derive the aerodynamic pressure from the linearized potential equation. Galerkin method was used to transform the governing partial differential equation to a series of ordinary differential equations. Based on Melnikov's method, the chaotic behaviors of the panel system were analyzed and suppressed by adding a parametric excitation term to the chaotic system. The numerical results illustrating the feasibility of the method are presented as bifurcation diagrams, phase plots, Poincaré maps, time-history diagrams and maximum Lyapunov exponent diagrams. The method used in this paper leads to fully consistent results. Comparison between the first-order and four-order expansion shows that the first-order expansion can qualitatively reflect the dynamical features of the plate.
\end{abstract}

Keywords Chaos suppression - Melnikov's method · Subsonic flow $\cdot$ External excitation $\cdot$ Thin panel

\section{Introduction}

The speed of trains has rapidly increased and a trend towards higher train speeds has evolved in recent decades. The aeroelastic problems accompanying by the speed-up of train systems are receiving a lot of attention as practical engineering issues that require urgent resolution $[1,2]$. Because streamlined design of high-speed trains is the general design

H. Tang · P. Li $(\bowtie) \cdot$ Y. Yang

School of Mechanics and Engineering, Southwest Jiaotong University, Chengdu 610031, People's Republic of China

e-mail: meiyongyuandeze@163.com to decrease running resistance, lots of panel structures such as the train body skin panels are used. The external panels of high-speed train traveling at high speed through air can be severely excited by the aerodynamic pressure. Moreover, the panels are also excited by the external forcings transferred from the wheel-rail excitation. In the test of the WuhanGuangzhou railway passenger dedicated line of China, the train body skin panels and windows vibrated obviously and caused strong noise radiation when the CRH-3 high-speed trains moved at almost $400 \mathrm{~km} / \mathrm{h}$. The aerodynamic and external forcings induced vibrations, especially chaotic vibrations, could inflict fatigue damage on the panel structures. Such problems must be taken into consideration in designing highspeed trains to have a considerable service. One of the most important issues of the panel flow-induced vibration problems is to predict the chaos and then suppress and control it.

Research on the complicated dynamic behaviors of thin panel has received much attention. Everall and Hunt [3] investigated the stability of a post-buckled, simply supported thin panel under compression for different in-panel boundary conditions. The dynamics of a post-buckling thin panel were studied by using the finite element method [4]. The nonlinear dynamics and bifurcations of orthotropic circular panels with simply supported boundary condition were analyzed by Akour and Nayfeh [5]. Awrejcewicz et al. [6] used the Bubnov-Galerkin approach with high-order approximations and a finite difference method to investigate the nonlinear vibrations and chaotic dynamics of a transversally and axially excited thin plate. Zhang et al. [7] investigated the bifurcation and Shilnikov type chaotic dynamics with both parametric and external excitations. Dowell [8] pointed out that the aeroelasticity of panel is a rich source of static and dynamic instability and chaotic motions.

Currently, the running speed of high-speed trains is about $350 \mathrm{~km} / \mathrm{h}$, which corresponds to a Mach number of about 
0.3 and is in the low-subsonic range basically. The instability and dynamics behaviors of thin panel in subsonic flow have been studied. Dugundji et al. [9] noted that the simply supported panel on continuous elastic foundations could flutter at a low Mach number of 0.125 , which was proved by the wind tunnel test. Kornecki et al. [10,11] used two theoretical approaches to model the flow around an elastic plate and Galerkin method was applied to investigate the instability of panels with different boundary conditions. Recently, Li et al. [12,13] analyzed the dynamical characteristics of a nonlinear thin panel subjected to subsonic flow and external excitations; they noted that the subsonic panel with external excitation displays a rich source of complex dynamics, such as period-doubling bifurcations, chaotic motions, and transient chaos with complex period windows. However, the nonlinearity in their model is less realistic [13], moreover, the authors do not imply suppression of the panel chaotic motions in their paper and it is a main shortcoming and need to be improved [12].

Chaotic system is a very complex nonlinear dynamical system. One of the basic characteristics of chaos is the repellency of any two adjacent trajectories of chaos, which results in a positive top Lyapunov exponent of the system. The chaos control for engineering application has emerged as a new and important topic. In 1990, Ott et al. [14] showed that a chaotic attractor could be converted to any one of a large number of possible attracting time-periodic motions by making only small time-dependent parameter perturbation. This method is called OGY method and it is a kind of feedback control [14-17]. Feedback control methods are used to control chaos by stabilizing a desired unstable periodic solution which is embedded in chaotic attractors. Another kind of chaos control is the non-feedback control [18-21], which is realized by adding some meaningful parameters, such as the external excitations [22]. So far, both two kinds of chaos control methods are still in development.
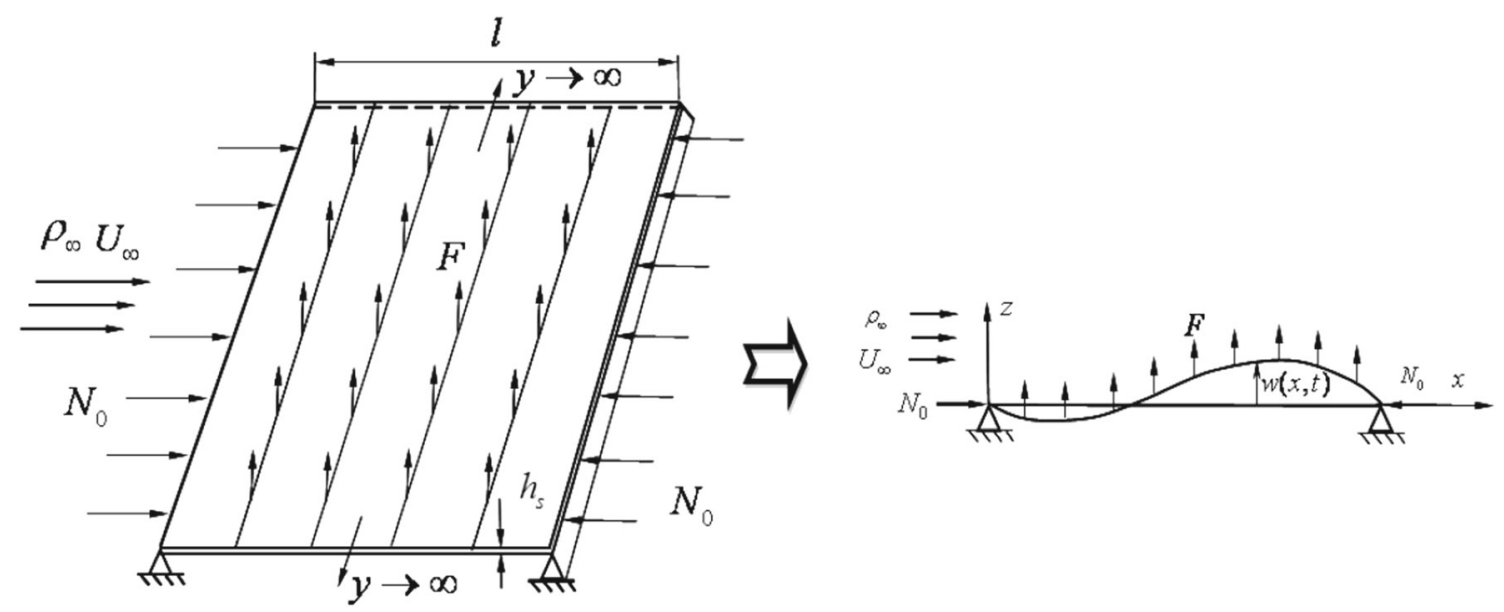

Fig. 1 Sketch of a two-dimensional thin panel in subsonic flow with external excitation 
where $w, \rho_{s}, h_{s}, E, v, g_{s}$ denote the bending deflection, mass density, thickness, elastic modulus, Poisson ratio and structure damping coefficient of the panel, respectively. $D=E h_{s}^{3} /\left[12\left(1-v^{2}\right)\right]$ is the panel bending stiffness. $P_{a}$ is the aerodynamic pressure (positive downwards as shown is Fig. 1).

The boundary conditions for a simply supported panel are:

$\left.w\right|_{x=0, l}=0,\left.\frac{\partial^{2} w}{\partial x^{2}}\right|_{x=0, l}=0$.

The aerodynamic pressure $P_{a}$ is in general given by the form (see ref. [23]):

$P_{a}=-\left.\rho_{\infty}\left(\frac{\partial \varphi}{\partial t}+U_{\infty} \frac{\partial \varphi}{\partial x}\right)\right|_{z=0} 0 \leq x \leq l$.

where $\rho_{\infty}$ is the air density, $U_{\infty}$ is the main flow velocity, and $\varphi$ is the velocity perturbation potential.

The velocity perturbation potential must satisfy the equation:

$\chi^{2} \frac{\partial^{2} \varphi}{\partial x^{2}}+\frac{\partial^{2} \varphi}{\partial z^{2}}=0$

where $\chi^{2}=1-M_{\infty}^{2}$ and $M_{\infty}$ is the flow Mach number, for subsonic flow, $M_{\infty}^{2}<1$.

Equation (4) must be solved for $\varphi$ subject to appropriate boundary conditions. In the linear approximation for a thin panel (see ref. [23]), there are:

$\left.\frac{\partial \varphi}{\partial z}\right|_{z=0}=\left(\frac{\partial w}{\partial t}+U_{\infty} \frac{\partial w}{\partial x}\right) \quad 0 \leq x \leq l$.

A Galerkin-type solution will be used for Eq. (1), assuming:

$w(x, t)=\sum_{m=1}^{M} g_{m}(t) \sin \left(\frac{m \pi x}{l}\right)$.

The assuming modes satisfy the boundary conditions Eq. (2), and then Eq. (5) reduces to:

$$
\begin{aligned}
\left.\frac{\partial \varphi}{\partial z}\right|_{z=0}= & \sum_{m=1}^{M}\left[\frac{d g_{m}}{d t} \sin \left(\frac{m \pi x}{l}\right)\right. \\
& \left.+\frac{m \pi g_{m} U_{\infty}}{l} \cos \left(\frac{m \pi x}{l}\right)\right] \quad 0 \leq x \leq l .
\end{aligned}
$$

In view of the form of these boundary conditions, $\varphi$ may be expressed as follows [23]:

$$
\begin{aligned}
\varphi= & \sum_{m=1}^{M}\left[C_{m}(t) \sin \left(\frac{m \pi x}{l}\right)+D_{m}(t) \cos \left(\frac{m \pi x}{l}\right)\right] e^{-\chi \frac{m \pi z}{l}} \\
& 0 \leq x \leq l
\end{aligned}
$$

Equation (8) satisfies Eq. (4) naturally, and then applying the boundary condition, one obtains:

$C_{m}=-\frac{l}{\chi m \pi} \frac{d g_{m}}{d t} ; \quad D_{m}=-\frac{U_{\infty} g_{m}}{\chi}$.

Substituting this expression into Eqs. (8) and (3), one obtains:

$$
\begin{aligned}
P_{a}= & \sum_{m=1}^{M}\left[\left(\frac{\rho_{\infty} l}{m \pi \chi} \frac{d^{2} g_{m}}{d t^{2}}-\frac{m \rho_{\infty} U_{\infty}^{2} \pi g_{m}}{\chi l}\right) \sin \left(\frac{m \pi x}{l}\right)\right. \\
& \left.+2\left(\frac{\rho_{\infty} U_{\infty}}{\chi} \frac{d g_{m}}{d t}\right) \cos \left(\frac{m \pi x}{l}\right)\right] .
\end{aligned}
$$

Introduce the dimensionless parameters:

$$
\begin{aligned}
q_{m} & =\frac{g_{m}}{h_{s}}, \quad \eta=\frac{x}{l}, \quad \tau=\left(\frac{D}{\rho_{s} h_{s}}\right)^{1 / 2} \frac{t}{l^{2}}, \quad \mu=\frac{\rho_{\infty} l}{\rho_{s} h_{s} \chi \pi}, \\
\lambda & =\frac{\rho_{\infty} U_{\infty}^{2} l^{3}}{\chi D}, \\
\Omega & =\omega l^{2}\left(\frac{D}{\rho_{s} h_{s}}\right)^{-1 / 2}, \quad \zeta=\left(\frac{D}{\rho_{s} h_{s}}\right)^{1 / 2} \frac{g_{s}}{l^{2}}, \quad R_{0}=\frac{N_{0} l^{2}}{D}, \\
f & =\frac{F l^{4}}{D h_{s}} .
\end{aligned}
$$

Substituting Eqs. (6), (10), (11) into Eq. (1) and applying the Galerkin method results in the equation of the panel motion for the $m$ th mode coordinate in dimensionless form:

$$
\begin{aligned}
\left(1+\frac{\mu}{m}\right) \ddot{q}_{m}+\left[(m \pi)^{4}-R_{0}(m \pi)^{2}-\lambda(m \pi)\right] q_{m} \\
\quad+3(m \pi)^{2} q_{m} \sum_{r=1}^{M}(r \pi)^{2} q_{r}^{2} \\
\quad+6(m \pi)^{2} \zeta q_{m} \sum_{r=1}^{M}(r \pi)^{2} q_{r} \dot{q}_{r}+(m \pi)^{4} \zeta \dot{q}_{m} \\
\quad+4\left(\frac{\lambda \mu}{\pi}\right)^{\frac{1}{2}} \sum_{m \neq r, r=1}^{M} \frac{1-(-1)^{m+r}}{m^{2}-r^{2}} m \dot{q}_{r} \\
=\frac{2 f}{m \pi}\left[1-(-1)^{m}\right] \cos (\Omega \tau) \quad m=1,2, \ldots, M .
\end{aligned}
$$

\section{Chaotic behaviors}

\subsection{First-order expansion}

Consider $F(x)$ in Eq. (1) to be constant in the following discussion. Truncate Eq. (7) to the first term and let $(u, v)=(q, \dot{q})$, this is helpful to make the nature of subsonic panel aeroelastic mathematical model (Eq.12) as transparent as possible [24]. Hence the Eq. (12) can be rewritten as:

$$
\left\{\begin{array}{l}
\dot{u}=v \\
\dot{v}=-\alpha u-\beta u^{3}-\varepsilon \delta_{1} v-\varepsilon \delta_{2} u^{2} v+\varepsilon h \cos (\Omega \tau)
\end{array} .\right.
$$


where $\alpha=\left(\pi^{4}-\lambda \pi-R_{0} \pi^{2}\right) /(1+\mu), \beta=3 \pi^{4} /(1+\mu)$, $\varepsilon \delta_{1}=\pi^{4} \zeta /(1+\mu), \varepsilon \delta_{2}=6 \zeta \pi^{4} /(1+\mu), \varepsilon h=4 f / \pi(1+\mu)$.

If $\varepsilon=0$, which means there is no damping and external excitation in the system, Eq. (13) is regarded as an unperturbed system and can be written as:

$\left\{\begin{array}{l}\dot{u}=v \\ \dot{v}=-\alpha u-\beta u^{3} .\end{array}\right.$

The system (14) is a Hamiltonian system and with Hamiltonian function $H(u, v)=\frac{v^{2}}{2}+\frac{\alpha u^{2}}{2}+\frac{\beta u^{4}}{4}$ and the potential function is $V(u)=\frac{\alpha u^{2}}{2}+\frac{\beta u^{4}}{4}$.

The critical dynamic pressure is obtained by setting $\alpha=0$, which implies $\lambda_{c}=\pi^{3}-R_{0} \pi$. Then define the dynamic pressure increment $\Delta \lambda=\lambda-\lambda_{c}$ in the following discussion.

The critical flow velocity proposed by Eq. (11) is:

$U_{\infty}=\sqrt{\frac{b \sqrt{b^{2}+4\left(a_{\infty}\right)^{4}}-b^{2}}{2\left(a_{\infty}\right)^{2}}}$.

where $b=\frac{\lambda_{c} D}{\rho_{\infty} l^{3}}, a_{\infty}$ is the flow sound velocity.

In Kornecki et al. [11], it is proposed to compute the critical velocity using

$U_{\infty}=\sqrt{\frac{\lambda_{c} D}{\rho_{\infty} l^{3}}}$.

Taking the aluminum alloy skins widely used for high speed trains, the values of the panel basic parameters in Fig. 1 are taken to be $E=69 \mathrm{GPa}, l=800 \mathrm{~mm}, \rho_{s}=2,705 \mathrm{~kg} / \mathrm{m}^{3}$, $g_{s}=0.001 \mathrm{~s}, h=2.5 \mathrm{~mm}, v=0.3$ and let parameter $R_{0}=-10$, so critical aerodynamic pressure is $\lambda_{c}=62.442$ and $\varepsilon \delta_{1}=0.9321, \beta=279.6433, \varepsilon \delta_{2}=3.7284$. The corresponding critical flow velocity proposed by Eq. (15) is $U_{\infty}=378.5923 \mathrm{~km} / \mathrm{h}$. When high speed trains are running, they may reach the critical velocity. The critical flow velocity proposed by Eq. (16) is found to be $U_{\infty}=388.2309 \mathrm{~km} / \mathrm{h}$. The critical flow velocity obtained in this paper is less than that proposed by Kornecki et al. [11]. The difference found here is due to the inclusion of the flow Mach number in the present paper, which is more realistic for high-speed trains.

The authors Li et al. [12] have used Melnikov's method to study the chaos of system (13), and some basic results for the unperturbed system (14) can be obtained directly without any difficulties.

Result 1. When $\Delta \lambda<0$, there is only one fixed points: $C(0,0)$ being center.

Result 2. When $\Delta \lambda>0$, there is a hyperbolic saddle point: $S(0,0)$ and $C_{i}(i=1,2)$ being centers $( \pm \sqrt{-\alpha / \beta}, 0)$. The saddle is connected to itself by two homoclinic orbits:

$q_{0}^{ \pm}(\tau)=\left(u_{0}^{ \pm}, v_{0}^{ \pm}\right)= \pm(\sqrt{-2 \alpha / \beta} \sec h(\sqrt{-\alpha} \tau), \alpha \sqrt{2 / \beta}$ $\sec h(\sqrt{-\alpha} \tau) \operatorname{th}(\sqrt{-\alpha} \tau))$, and these orbits satisfy $u_{0}^{ \pm}(\tau)=$ $u_{0}^{ \pm}(-\tau), v_{0}^{ \pm}(\tau)=-v_{0}^{ \pm}(-\tau)$.
The Melnikov's functions for these homoclinic orbits are:

$$
\begin{aligned}
M_{ \pm}\left(\tau_{0}\right)= & \int_{-\infty}^{+\infty} v_{0}^{ \pm}(\tau)\left(-\delta_{1} v_{0}^{ \pm}(\tau)-\delta_{2}\left(u_{0}^{ \pm}(\tau)\right)^{2} v_{0}^{ \pm}(\tau)\right. \\
& +h \cos \left(\Omega\left(\tau+\tau_{0}\right)\right) d \tau \\
= & -\delta_{1} I_{1}-\delta_{2} I_{2} \pm h I_{3} \sin \left(\Omega \tau_{0}\right)
\end{aligned}
$$

where $I_{1}=\frac{4}{3} \frac{(-\alpha)^{\frac{3}{2}}}{\beta}, I_{2}=\frac{16}{15} \frac{(-\alpha)^{\frac{5}{2}}}{\beta^{2}}, I_{3}(\Omega)=\sqrt{\frac{2}{\beta}} \Omega \pi$ $\sec h\left(\frac{\Omega \pi}{2 \sqrt{-\alpha}}\right)$.

A pitchfork-like bifurcation occurs with the increasing dynamic pressure. The number of the fixed points and their stabilities will change after the dynamic pressure exceeds the critical value $\lambda_{c}$. The potential functions and phase plots of system (14) for $\Delta \lambda<0$ and $\Delta \lambda>0$ are shown in Fig. 2. In Fig. 2b, it is referred to as a double-well potential, its two wells being separated by a potential barrier. The orbits that emerge from and converge to the saddle point are the homoclinic orbits $q_{0}^{ \pm}(\tau)$.

According to Result 2, obviously, if the parameters satisfy:

$h>\tilde{h}=\left|\frac{\delta_{1} I_{1}+\delta_{2} I_{2}}{I_{3}(\Omega)}\right|$.

then $M_{ \pm}\left(\tau_{0}\right)=0$ and $\partial M_{ \pm} / \partial t\left(\tau_{0}^{ \pm}\right) \neq 0$. If $M_{ \pm}\left(\tau_{0}\right)$ has simple zeros, the stable and unstable manifolds of the fixed points corresponding to periodic orbits may split and have transverse intersection under the weak periodic perturbation, and will result in chaos by the Smale-Birkhoff theorem $[25,26]$.

In fact, lots of numerical and experimental results show that there is another critical value denoted $h_{c}$ governing the occurrence of a chaotic behavior in the common sense and $h_{c}$ is always larger than $h$. Taking $\Delta \lambda=5.5(a=$ 16.5347), $\Omega=1.0$, the critical parameter for chaos proposed by Melnikov's method (Eq. 17) is $\varepsilon h=1.3285$. Then Eq. (13) is simulated by using the fourth order RungeKutta method. Numerical calculations have produced the bifurcation diagram of Fig. 3a, as the forcing amplitude $\varepsilon h$ varying in the range of $0.8 \leq \varepsilon h \leq 1.9$. The maximum Lyapunov exponent diagram corresponding to Fig. 3a is shown in Fig. $3 b$ to confirm the chaotic motions. Numerical results from Fig. 3 show that the critical parameter for occurrence of chaos is $\varepsilon h_{c}=1.552$. That is to say, if $\varepsilon h=\varepsilon h_{c}$ is adopted in system (13), chaos appear. The phase plot and corresponding Poincaré map are shown in Fig. 4 for $\varepsilon h_{c}=1.552$. It is seen that the Poincaré map exhibits dense iterate structure consistent with chaos. The critical parameters of chaos proposed by the numerical results are thus in an agreement with that proposed by Melnikov's method. 

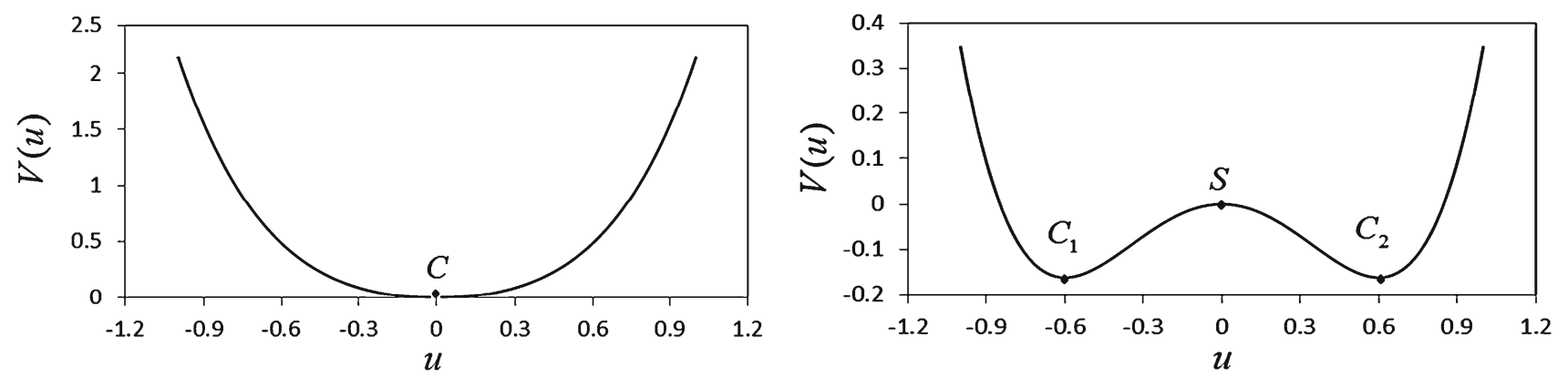

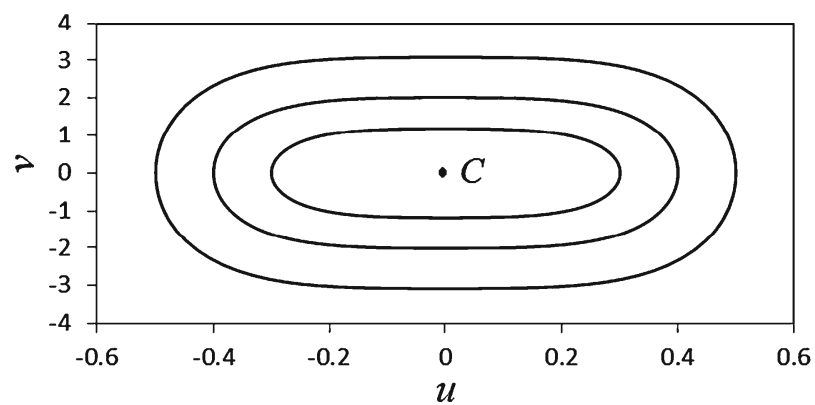

(a)

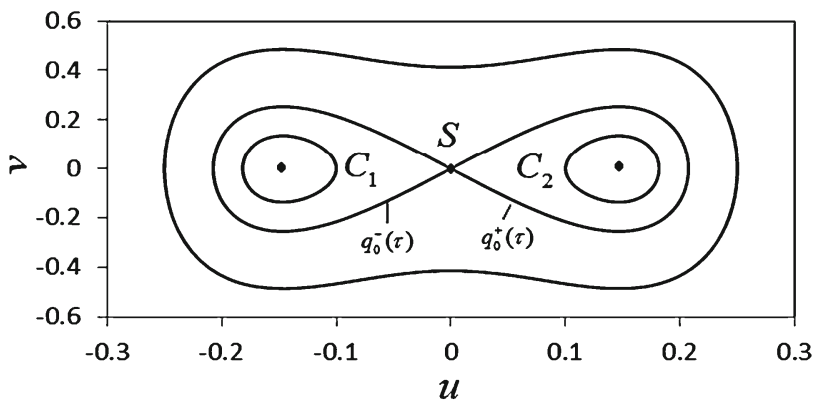

(b)

Fig. 2 Phase plots and potential plots of system (14): a $\Delta \lambda<0$, b $\Delta \lambda>0$
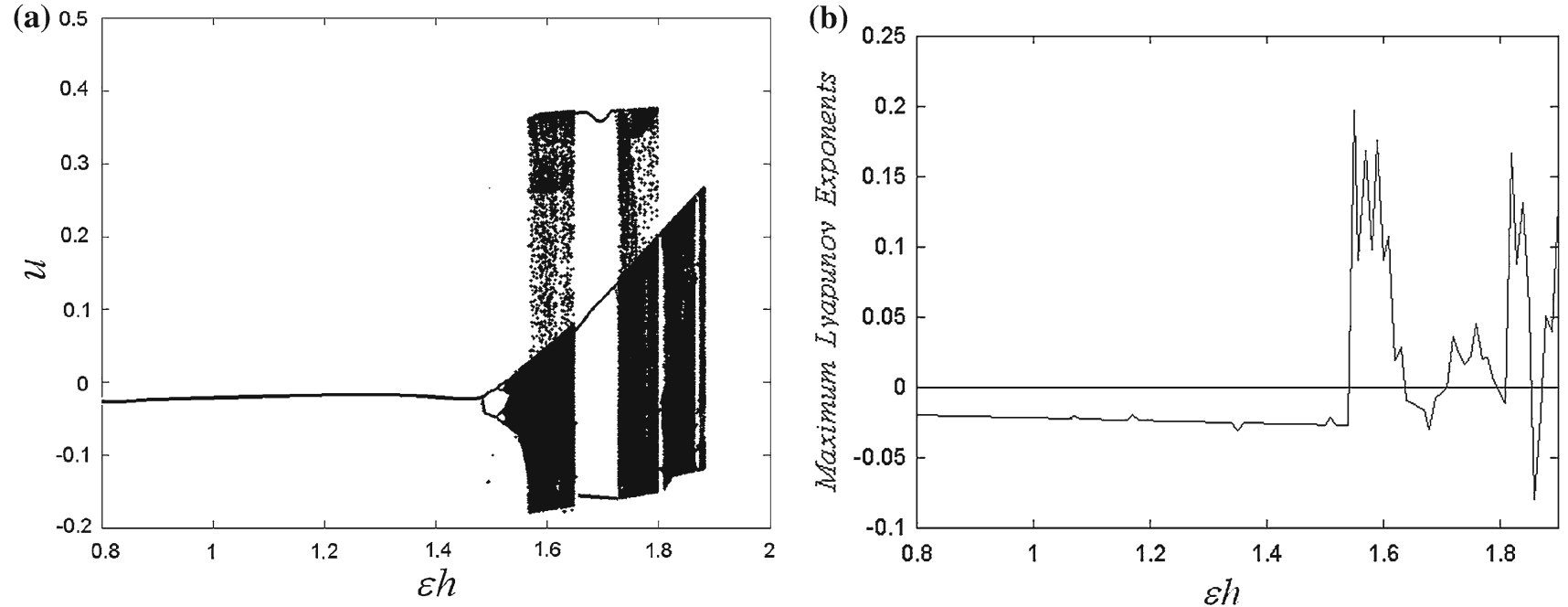

Fig. 3 Bifurcation diagram $(u-\varepsilon h)$ and maximum Lyapunov exponent diagram of system (13): a bifurcation diagram, $\mathbf{b}$ maximum Lyapunov exponent diagram

\subsection{Higher-order expansions}

When it comes to higher order expansion, Eq. 12 represents the equation of motion of the plate. Comparison of the motions of the plate between the first-order expansion and higher-order expansion is made in this subsection. The nonlinear dynamics of the system with four-order expansion is conducted numerically. Figure 5a shows the phase plots of the displacement at the center of the plate for the first order and first four order expansions for $\varepsilon h_{c}=2.0, \Delta \lambda=$ $-1.0, \Omega=1.0$. And the time-history diagrams at the center of the plate for $\varepsilon h_{c}=1.552, \Delta \lambda=5.5, \Omega=1.0$ are shown in Fig. 5b. From Fig. 5, it is clear that both the results are periodic motions in Fig. 5a and chaotic motions in Fig. 5b. Although there are some differences between the first order and first four order expansions, the first order expansion can qualitatively show the dynamical features of the plate in subsonic flow. 

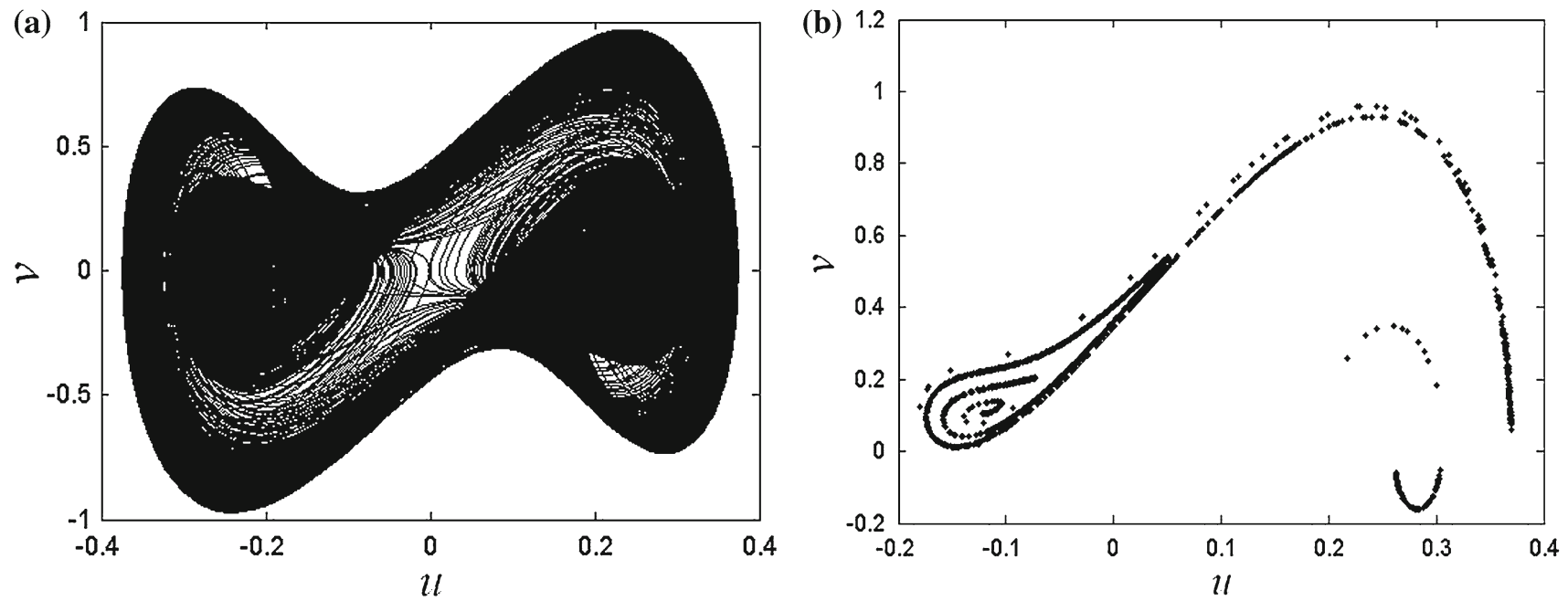

Fig. 4 Phase plots and Poincaré map of system (13) for $\varepsilon h=1.552$ : a phase plots, $\mathbf{b}$ Poincaré map
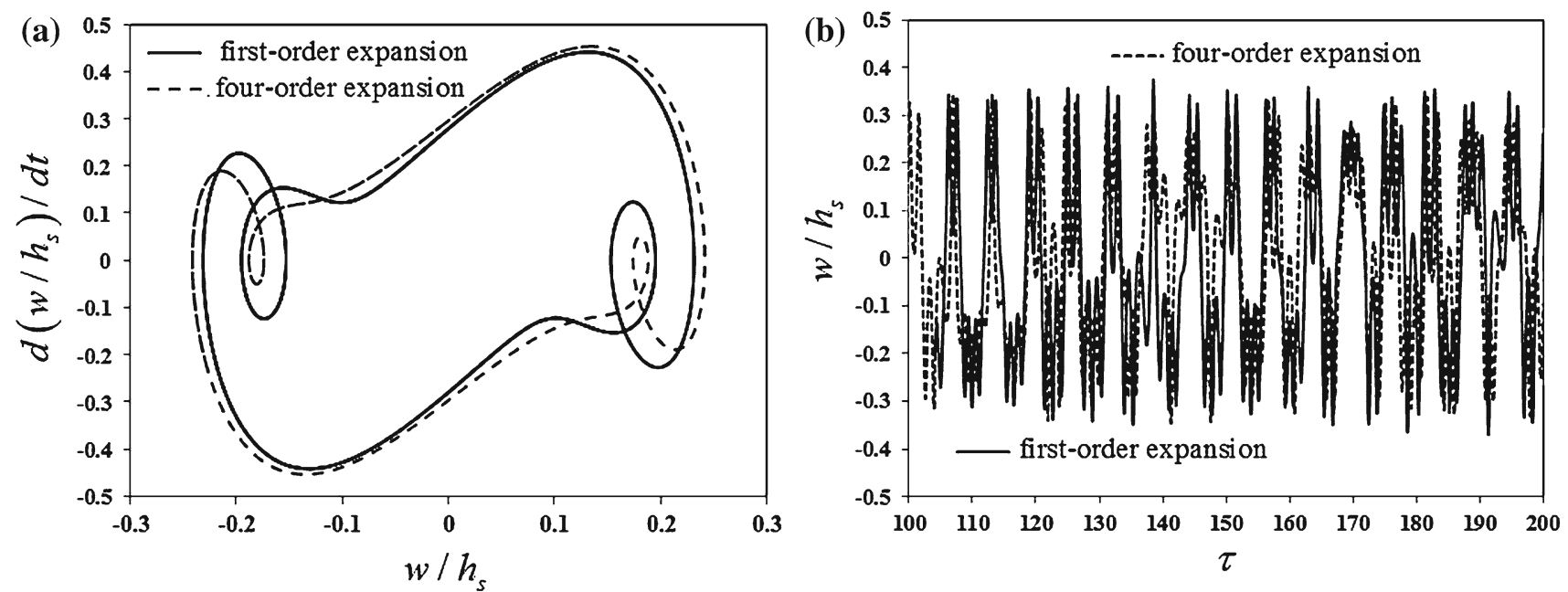

Fig. 5 The a phase plots and $\mathbf{b}$ time-history diagram of the displacement at the center of the plate

\section{Chaos suppression}

Here one considers the use of piezoelectric material to implement the suppression of panel chaotic motions, which has been widely used in the control of structure vibration. Piezoelectric patch was pasted on the bottom side of the panel as shown in Fig. 6, which can generate an excitation on the panel by applying an appropriate voltage $V_{a}$. The applied voltage is always determined by a suppression algorithm based on the input information (i.e., $h_{c}$ for chaotic motions appear). The algorithm is the key issues of applying chaos suppression.

According to the analysis in Sect. 3, the existence of simple zeros of the Melnikov's function is a necessary condition to determine chaos in the common sense. If by adding some meaningful terms to the chaotic system, such that the Melnikov's function of the new system has no simple zeros, then

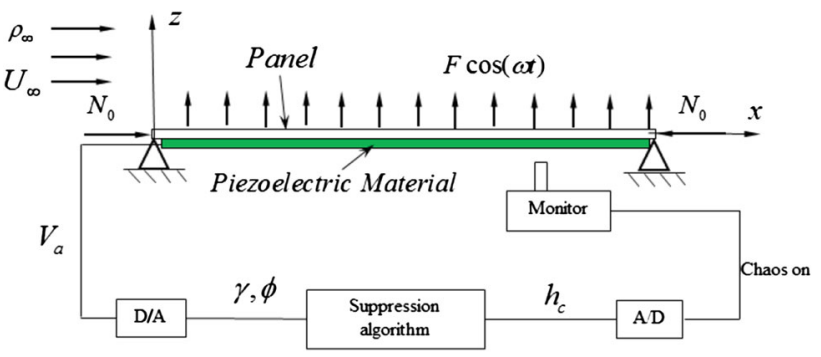

Fig. 6 Block diagram of panel chaos suppression

chaotic motions are suppressed. This implement is realized by adding a parametric excitation term to the chaotic system (see Ref. [22]), which is a very simple and easy to implement and has been used in the chaotic dynamical system [27]. 
Adding a parameters term to chaotic system, now the new system is:

$\left\{\begin{array}{rl}\dot{u}= & v \\ \dot{v}= & -\alpha u-\beta u^{3}-\varepsilon \delta_{1} v-\varepsilon \delta_{2} u^{2} v \\ & +\varepsilon h_{c} \cos (\Omega \tau)+\varepsilon \gamma h_{c} \cos (\Omega \tau+\phi)\end{array}\right.$.

where $\varepsilon \gamma h_{c} \cos (\Omega \tau+\phi)$ is an added parametric excitation; $\gamma, \phi$ are the amplitude and phase of the parametric term and $\phi$ is considered as in the range of $-\frac{\pi}{2} \leq \phi \leq \frac{\pi}{2}$.

The Melnikov's function of Eq. (18) is:

$$
\begin{aligned}
M_{ \pm}\left(\tau_{0}\right)= & \int_{-\infty}^{+\infty} v_{0}^{ \pm}(\tau)\left(-\delta_{1} v_{0}^{ \pm}(\tau)-\delta_{2}\left(u_{0}^{ \pm}(\tau)\right)^{2} v_{0}^{ \pm}(\tau)\right. \\
& +h_{c} \cos \left(\Omega\left(\tau+\tau_{0}\right)\right)
\end{aligned}
$$

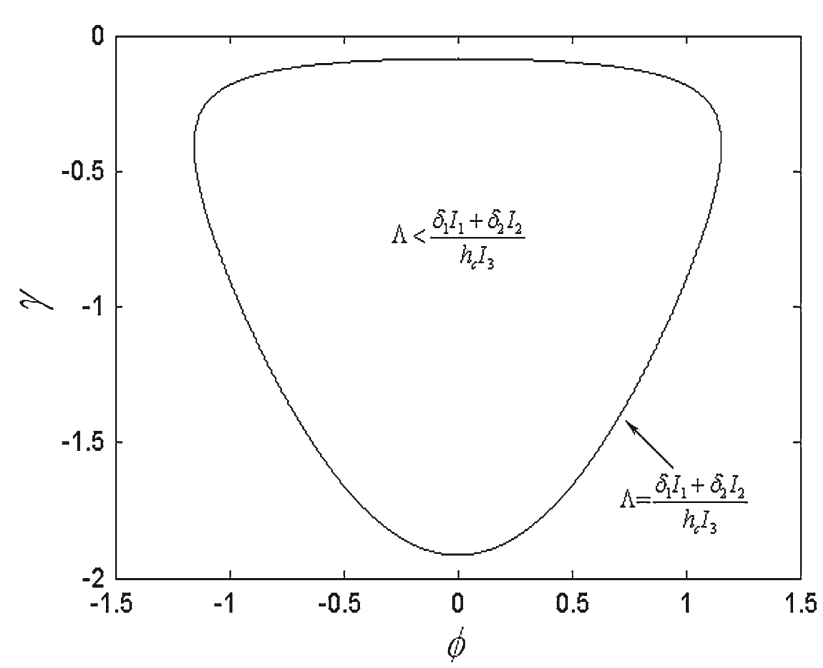

Fig. 7 The critical curve of parameters $\gamma$ and $\phi$

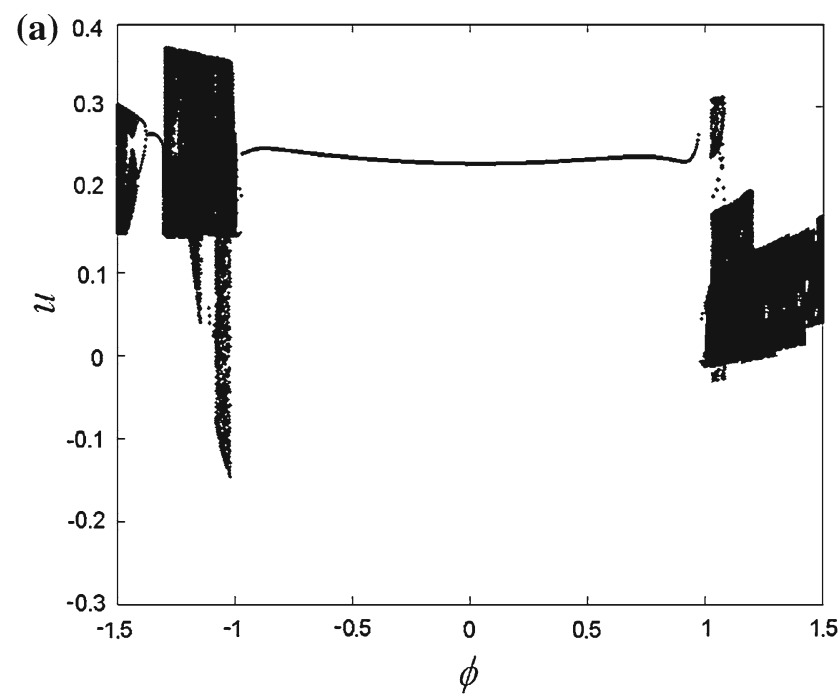

$$
\begin{aligned}
& \left.+\gamma h_{c} \cos \left(\Omega\left(\tau+\tau_{0}\right)+\phi\right)\right) d \tau \\
= & -\delta_{1} I_{1}-\delta_{2} I_{2} \pm h_{c} I_{3} \sin \left(\Omega \tau_{0}\right) \\
& \pm \gamma h_{c} I_{3} \sin \left(\Omega \tau_{0}+\phi\right) \\
= & -\delta_{1} I_{1}-\delta_{2} I_{2} \pm h_{c} I_{3}\left[(1+\gamma \cos \phi) \sin \left(\Omega \tau_{0}\right)\right. \\
& \left.+\gamma \sin \phi \cos \left(\Omega \tau_{0}\right)\right] \\
= & -\delta_{1} I_{1}-\delta_{2} I_{2} \pm h_{c} I_{3} \Lambda \sin \left(\Omega \tau_{0}+\psi\right) .
\end{aligned}
$$

where

$\Lambda=\sqrt{1+2 \gamma \cos \phi+\gamma^{2}}, \quad \sin \psi=\frac{\gamma \sin \phi}{\Lambda}$,

$\cos \psi=\frac{1+\gamma \cos \phi}{\Lambda}$.

When $h_{c} I_{3} \Lambda<\delta_{1} I_{1}+\delta_{2} I_{2}$, that is

$\Lambda<\left|\frac{\delta_{1} I_{1}+\delta_{2} I_{2}}{h_{c} I_{3}(\Omega)}\right|$.

then $M_{ \pm}\left(\tau_{0}\right)$ has no zeros thus the chaos of the chaotic system is suppressed.

The critical parameter curve $(\gamma-\phi)$ of Eq. (20) is shown in Fig. 7. It shows that the critical curve is symmetric with the line $\phi=0$, and the range of $\phi$ firstly increases and then decreases with the decreasing $\gamma$. If the parameters $\gamma$ and $\phi$ in Eq. (20) take the values of the inside points enclosed by the curve in Fig. 7, the chaos is suppressed. These points can be randomly selected.

The bifurcation diagrams and corresponding maximum Lyapunov exponent diagrams of the parameters $\phi$ and $\gamma$ are shown in Figs. 8 and 9, respectively. The bifurcation diagram $(u-\phi)$ for $\gamma=-1$ is shown in Fig. 8 and the phase $\phi$ varies in the range of $-1.5 \leq \phi \leq 1.5$. From these figures, one can see that when $\phi$ is in the range of $-0.98 \leq \phi \leq 0.98$, the motion is periodic, and chaos is suppressed. From Fig. 9, one knows

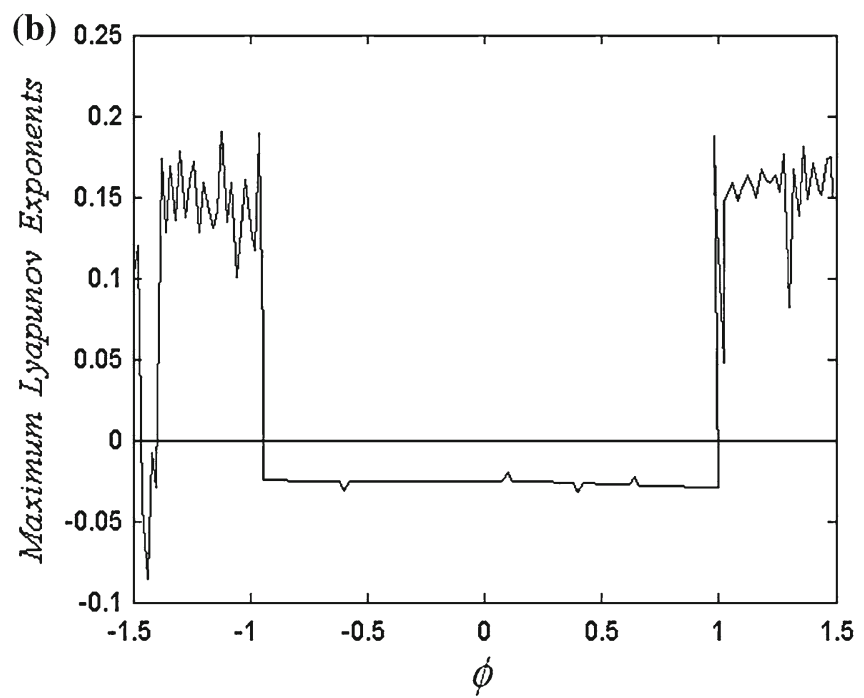

Fig. 8 Bifurcation diagram $(u-\phi)$ and maximum Lyapunov exponent diagram of system (18) for $\gamma=-1$ : a bifurcation diagram, $\mathbf{b}$ maximum Lyapunov exponent diagram 

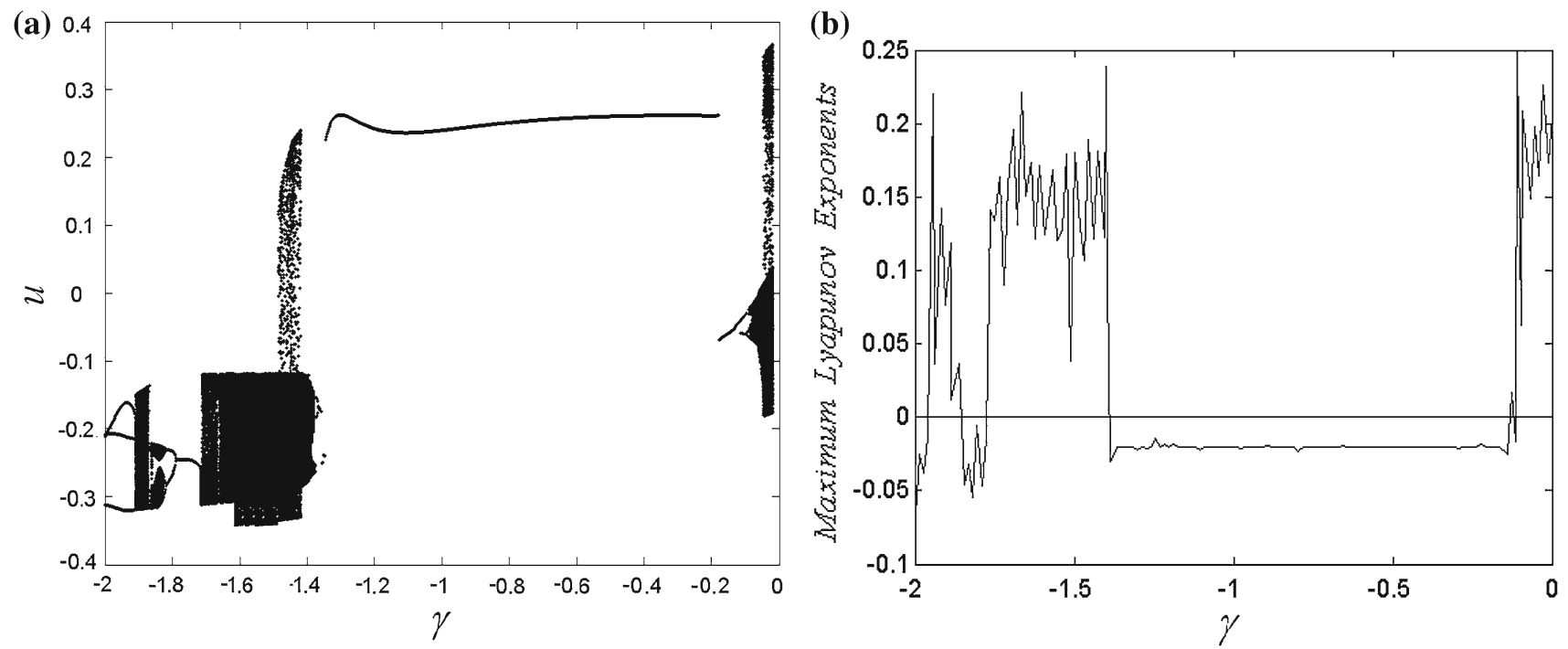

Fig. 9 Bifurcation diagram $(u-\gamma)$ and maximum Lyapunov exponent diagram of system (18) for $\phi=0.75$ : a bifurcation diagram, b maximum Lyapunov exponent diagram
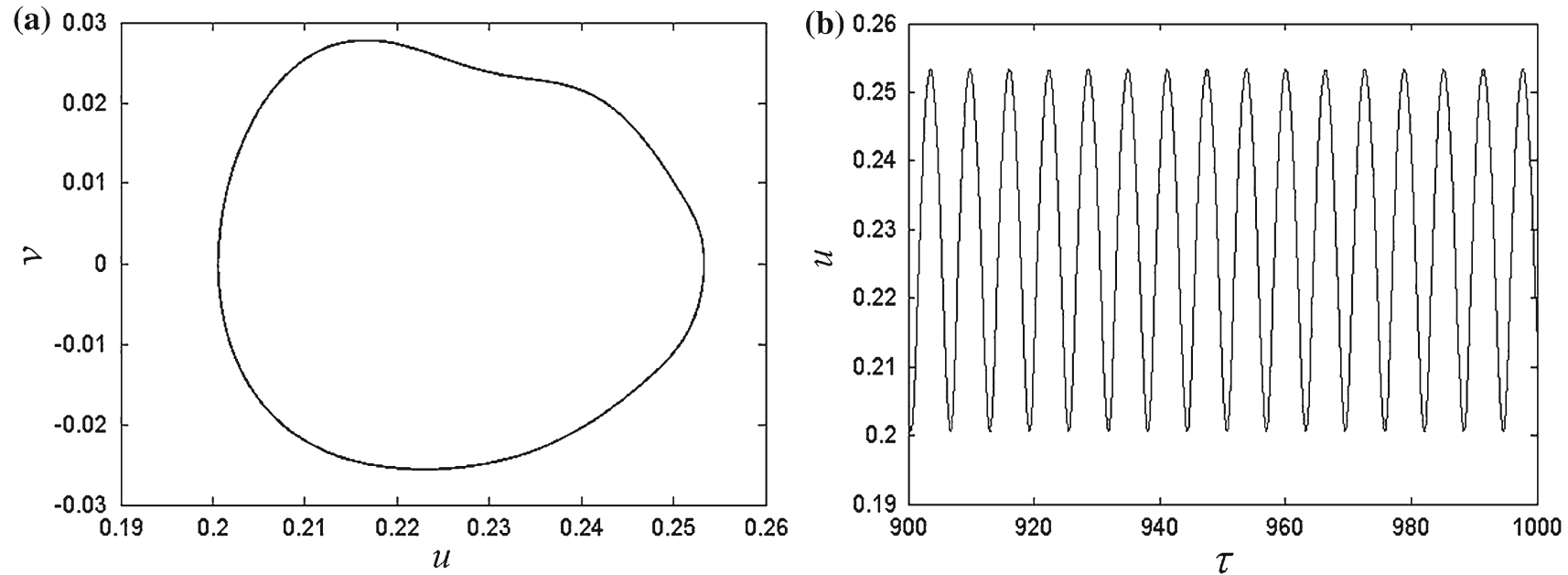

Fig. 10 The periodic solution after control for $\gamma=-1, \phi=0.5$ (the same parameters and initial conditions as in Fig. 4): a phase plot, b time-history diagram

that when the parameter $\gamma$ varies in the range of $-1.38 \leq \phi \leq$ -0.12 , the chaotic motion changes to periodic motion and chaotic motion is suppressed. The same conclusions can also be reached from these corresponding maximum Lyapunov exponent diagrams (see Figs. 8b and 9b).

Figure 10 shows the phase plot and time-history diagram after suppression (note the difference between Fig. 4 before suppression and Fig. 10 after suppression). From these figures, it is seen that the chaotic motion changes to the regular motion characterized by period-1 motion, and chaos suppression is realized. All the numerical results illustrate the validity of this method suggested in this paper.

\section{Summary}

The chaos suppression of a two-dimensional subsonic panel subjected to external excitation is analyzed based on Melnikov's method. For the unperturbed system, the number and stability of the fixed points change after the dynamic pressure exceeds the critical value, and then the homoclinic orbits appear. The critical parameters for chaos are determined as a function of the aerodynamic pressure, amplitude and frequency of external forcing. Based on Melnikov's method, the chaos of the system is suppressed by adding a parameters term to the chaotic system. Numerical results show an agreement with the theoretical analysis. The use of Melnikov's 
method for chaos of the subsonic panel subjected to external excitation presented in this paper is reasonable. The firstorder expansion can qualitatively reflect the dynamical characters of the plate.

It should be pointed out that only one-mode Galerkin reduction is considered in this paper, and the higher coupled modes are not involved. The authors are carrying on the wind tunnel experiments of panel chaos suppression and further researches on two-coupled modes reduction which results in two coupled periodically driven equations by using the method suggested in this paper. Some qualitative conclusions obtained in this paper can provide important referential value, especially for the wind tunnel experiments.

Acknowledgments The authors acknowledge the National Natural Science Foundation of China (Grant nos. 11302183 and 11072204), the Fundamental Research Funds for Central Universities (Grant No: 2682013CX026) and Sichuan Provincial Youth Science and Technology Innovation Team (2013) for financial support of this research. The authors are grateful to the reviewers and the authors of the references whose previous works helped greatly in writing this paper.

\section{References}

1. Schetz JA (2001) Aerodynamics of high-speed trains. Ann Rev Fluid Mech 33:371-414

2. Raghunathan RS, Kim HD, Setoguchi T (2002) Aerodynamics of high-speed railway trains. Prog Aerosp Sci 38:469-514

3. Everall PR, Hunt GW (1999) Arnold tongue predictions of secondary buckling in thin elastic plate. J Mech Phys Solids 47:21872206

4. Zhang W, Liu ZM, Yu P (2001) Global dynamic of a parametrically and externally excited thin plate. Nonlinear Dyn 24:245-268

5. Akour SN, Nayfeh JF (2006) Nonlinear dynamics of polarorthotropic circular plates. Int J Struct Stab Dyn 6:253-268

6. Awrejcewicz J, Krysko VA, Narkaitis GG (2003) Bifurcations of a thin plate-strip excited transversally and axially. Nonlinear Dyn 32:187-209

7. Zhang W, Zhang JH, Yao MH (2010) The extended Melnikov method for non-autonomous nonlinear dynamic systems and application to multi-pulse chaotic dynamic of a buckled thin plate. Nonlinear Anal 11:1442-1457

8. Dowell EH (1982) Flutter of a buckled plate as an example of chaotic motion of a deterministic autonomous system. J Sound Vib 85:333-344
9. Dugundji J, Dowell EH, Perkin B (1963) Subsonic flutter of panels on continuous elastic foundations. AIAA J 5:1146-1154

10. Kornecki A (1974) Static and dynamic instability of panels and cylindrical shells in subsonic potential flow. J Sound Vib 32:251263

11. Kornecki A, Dowell EH, O'Brien J (1974) On the aeroelastic instability of two-dimensional panels in unform incompressible flow. $\mathrm{J}$ Sound Vib 47:163-178

12. Li P, Yang YR, Zhang ML (2011) Melnikov's method for chaos of a two-dimensional thin panel in subsonic flow with external excitation. Mech Res Commun 38:524-528

13. Li P, Yang YR, Xu W (2012) Nonlinear dynamics analysis of a twodimensional thin panel with an external forcing in incompressible subsonic flow. Nonlinear Dyn 67:2483-2503

14. Ott E, Grebogi C, Yorke JA (1990) Controlling chaos. Phys Rev Lett 64:1196-1199

15. Shinbrot T, Ott E, Grebogi C, Yorke JA (1993) Using small perturbations to control chaos. Nature 363:411-417

16. Shinbrot T, Ott E, Grebogi C, Yorke JA (1990) Using chaos to direct trajectories. Phys Rev Lett 65:3215-3218

17. Lazzouni SA, Bowong S, Moukam Kakmeni FM, Cherki B, Ghouali N (2007) Chaos control using small-amplitude damping signal of the extend Duffing equation. Commun Nonlinear Sci Numer Simul 12:804-813

18. Ramesh M, Narayanan S (1990) Chaos control by non-feedback methods in the presence of noise. Chaos Solitons Fractal 10:14731489

19. Lima R, Pettini M (1990) Suppression of chaos by resonant parametric perturbations. Phys Rev Lett A1:726-733

20. Braiman Y, Goldhirsh I (1991) Taming chaotic dynamics with weak periodic perturbations. Phys Rev Lett 20:2545-2548

21. Mahmoud GM, Mohamed AA, Aly SA (2001) Strange attractors and chaos control in periodically forced complex Duffing's oscillators. Phys A 292:193-206

22. Li R, Xu W, Li S (2006) Chaos controlling of extended nonlinear Liénard system based on the Melnikov theory. Appl Math Comput 178:405-411

23. Dowell EH (1975) Aeroelasticity of plates and shells. Noordhoff International Publishing, Leyden

24. Li P, Yang YR, Xu W, Chen G (2013) Stochastic analysis of a nonlinear forced panel in subsonic flow with random pressure fluctuations. J Appl Mech 80:041005. doi:10.1115/1.4007819

25. Wiggins $S$ (1988) Introduction to applied nonlinear dynamical system and chaos. Springer, New York

26. Guckenheimer J, Holmes P (1985) Nonlinear oscillations, dynamical system, and bifurcation of Vector fields. Springer, New York

27. Wang RQ, Deng J, Jing ZJ (2006) Chaos control in doffing system. Chaos Solitons Fractal 27:249-257 